\title{
Dysregulated long non-coding RNAs in pleomorphic adenoma tissues of pleomorphic adenoma gene 1 transgenic mice
}

\author{
WANLIN XU ${ }^{1,2^{*}}$, LIMIN LIU ${ }^{2,3^{*}}$, HAO LU ${ }^{1,2}$, JINYE FU ${ }^{1,2}$, CHENPING ZHANG $^{1,2}$, \\ WENJUN YANG ${ }^{1,2}$ and SHUKUN SHEN ${ }^{1,2}$
}

\author{
${ }^{1}$ Department of Oral and Maxillofacial-Head and Neck Oncology, Shanghai Ninth People's Hospital, \\ College of Stomatology, Shanghai Jiao Tong University School of Medicine; ${ }^{2}$ National Clinical Research Center for \\ Oral Diseases, Shanghai Key Laboratory of Stomatology and Shanghai Research Institute of Stomatology; \\ ${ }^{3}$ Department of Oral Pathology, Shanghai Ninth People's Hospital, College of Stomatology, \\ Shanghai Jiao Tong University School of Medicine, Shanghai 200011, P.R. China
}

Received September 9, 2018; Accepted April 4, 2019

DOI: $10.3892 / \mathrm{mmr} .2019 .10149$

\begin{abstract}
Long non-coding RNAs (IncRNAs) have been proven to serve vital roles in various human diseases. However, their involvement in the development of pleomorphic adenoma (PA) in the salivary gland has yet to be examined. In the present study, microarray analysis of the IncRNA and mRNA expression profiles in pleomorphic adenoma gene 1 (PLAG1) transgenic mice was performed. Next, bioinformatics tools were used to predict the differentially expressed genes associated with PA, including Gene Ontology (GO), Kyoto Encyclopedia of Genes and Genomes (KEGG) pathway enrichment and IncRNA-mRNA co-expression network analyses. Comparison of the transgenic and control mice demonstrated that a total of 9,110 lncRNAs and 7,750 mRNAs were significantly differentially expressed (fold change $>2 ; \mathrm{P}<0.05$ ). Subsequently, six lncRNAs were randomly selected for further analysis, and five of these were validated as differentially expressed in PA by quantitative polymerase chain reaction, supporting the methodology employed in the current study. The GO and KEGG enrichment analysis of the differentially expressed mRNAs revealed that these mRNAs were closely associated with a number of processes involved in the development of PA. Furthermore, the IncRNA-mRNA co-expression network indicated that certain IncRNAs may serve vital roles in the pathogenesis of PA by interacting with a number of core
\end{abstract}

Correspondence to: Dr Wenjun Yang or Dr Shukun Shen, Department of Oral and Maxillofacial-Head and Neck Oncology, Shanghai Ninth People's Hospital, College of Stomatology, Shanghai Jiao Tong University School of Medicine, 639 Zhizaoju Road, Huangpu, Shanghai 200011, P.R. China

E-mail: ywjdoctor2@sina.com

E-mail: 450907991@qq.com

${ }^{*}$ Contributed equally

Key words: long non-coding RNAs, pleomorphic adenoma gene 1, transgenic mice, pleomorphic adenoma, microarray analysis genes. Taken together, these results indicated that lncRNAs and mRNAs were differentially expressed in PA tissues obtained from PLAG1 transgenic mice as compared with those from control mice. These differentially expressed lncRNAs may act as novel biomarkers and therapeutic targets for PA.

\section{Introduction}

Neoplasms of the salivary glands constitute 2-10\% of all head and neck tumors $(1,2)$. Among these, pleomorphic adenoma (PA), also known as 'mixed tumor', is one of the most common types of benign neoplasm of the salivary gland (3). PA presents as a slow-growing, painless and multi-nodular tumor that most frequently occurs in the parotid gland and less often in the submandibular gland, while it rarely occurs in the sublingual gland. The standard treatment for PA is extra-capsular excision of the tumor with or without partial excision of the affected gland. However, partial PA may recur or transform into carcinoma ex PA following surgery, which can lead to mortality in these patients (4).

Pleomorphic adenoma gene 1 (PLAG1), located on chromosome 8q12, is best known for its involvement in the development of various human tumors, such as lipoblastoma and hepatoblastoma $(5,6)$. Targeted disruption of PLAG1 may cause fetal growth restriction, and reduce sperm output and motility $(7,8)$. In salivary gland tumors, PLAG1 was initially identified as an oncogene associated with PA (9-11). More recently, it was demonstrated that this gene is also associated with carcinoma ex PA $(12,13)$. In our previous studies, PLAG1 transgenic mice were established, whose submandibular PA resembles the salivary gland PA of humans (14-16).

Long non-coding RNAs (lncRNAs) are a class of regulatory molecules that consist of $>200$ nucleotides and are not translated into proteins. A variety of lncRNAs have been identified in human and mouse diseases using large-scale analyses of RNA sequence data $(17,18)$. However, the correlation between IncRNAs and salivary gland diseases has not yet been widely studied. The lncRNA LINC00473 is a downstream target and an important mediator of the CRTC1-MAML2 fusion oncoprotein, which represents a promising biomarker 
and therapeutic target for human CRTC1-MAML2-positive mucoepidermoid carcinoma (19). Numerous lncRNA transcripts have been reported to be dysregulated in the labial salivary glands of patients with primary Sjögren's syndrome, which may serve important roles in the pathogenesis of this disease (20). These previous studies have indicated the crucial role of lncRNAs in salivary gland diseases. However, the expression of lncRNAs in PA remains unclear.

To the best of our knowledge, the present study is the first to use an lncRNA microarray to characterize the expression profiles of lncRNAs and mRNAs in the PLAG1 transgenic murine model of PA. The results indicated that dysregulated lncRNAs may serve a vital role in the pathogenesis of PA.

\section{Materials and methods}

Animals and sample collection. All animal experiments were approved by the Ethics Committee of the Faculty of Medicine, Shanghai Jiao Tong University (Shanghai, China). The PLAG1 transgenic mice were generated as described in our previous studies $(14,15)$. Briefly, mouse mammary tumor virus-PLAG1 transgenic mice were maintained in C57BL/6 background. A total of three male transgenic mice (age, 12 weeks; weight, 25-30 g) were used for subsequent experiments. In addition, three male wild type mice (age, 12 weeks; weight, 25-30 g) were used as control group. All animals ere purchased from The Shanghai Slake Laboratory Animal Co., Ltd (Shanghai, China). All mice were housed in cages under standard conditions at $24^{\circ} \mathrm{C}$ under a 12 -h light/dark cycle with a relative humidity of $55 \%$. All mice had free access to food and water. Submandibular tumors (average diameter, $1.5 \mathrm{~cm}$ ) from 3-month-old transgenic mice and normal submandibular glands from wild-type mice were collected. Three pairs of mouse tumors and control glands were divided into two groups, the tumor group, including mouse tumor 1 (MT1), MT2 and MT3 and the control group (Con1, Con 2 and Con3). These samples were analyzed independently for the expression of IncRNAs and mRNAs. Another six pairs of mouse tumors and control glands were used for subsequent polymerase chain reaction (PCR) detection.

RNA extraction. Total RNA was extracted from the mouse tumors and control glands using TRIzol ${ }^{\circledR}$ reagent (Thermo Fisher Scientific, Inc., Waltham, MA, USA). RNA quantity and quality were measured using a NanoDrop ND-1000 spectrophotometer (Thermo Fisher Scientific, Inc.). RNA integrity was assessed by standard denaturing agarose gel electrophoresis.

Microarray analysis. Arraystar Mouse LncRNA Microarray V3.0 (Arraystar, Inc., Rockville, MD, USA) is designed for the global profiling of mouse lncRNAs and protein-coding transcripts. Following RNA labeling and array hybridization, the microarray was scanned using the Agilent Microarray Scanner (Agilent p/n G2565BA; Agilent Technologies, Inc., Santa Clara, CA, USA). The Agilent Feature Extraction Software was used to extract data and for subsequent data processing. The lncRNA exhibiting a fold-change $\geq 2$ and $\mathrm{P}<0.05$ compared with the control were considered as differentially expressed.

Gene Ontology (GO) and Kyoto Encyclopedia of Genes and Genomes (KEGG) pathway enrichment analysis. GO analysis was conducted to identify the functions of differentially expressed genes, including biological processes, molecular functions and cellular components. Furthermore, the pathways associated with the differentially expressed mRNAs were assessed using KEGG analysis. Significant GO terms and pathways were selected on the basis of $\mathrm{P}<0.05$ and false discovery rate of $<0.05$. The unsupervised hierarchical clustering of the top 20 dysregulated lncRNAs and mRNAs was also performed.

Reverse transcription-quantitative PCR (RT-qPCR) validation. Subsequent to extraction, total RNA was reverse transcribed into cDNA using the PrimeScript RT Master Mix (Takara Biotechnology Co., Ltd., Dalian, China), according to the manufacturer's protocol. A total of four upregulated and two downregulated lncRNAs were selected for PCR validation on the basis of fold change (FC). The qPCR experiment was performed using SYBR Premix Ex Taq II (Takara Biotechnology Co., Ltd.) on the 7500 Sequence Detection system (ABI; Thermo Fisher Scientific, Inc.). The following thermocycling conditions were used: Initial denaturation at $95^{\circ} \mathrm{C}$ for $10 \mathrm{~min}$, followed by 40 cycles of $95^{\circ} \mathrm{C}$ for $10 \mathrm{sec}$ and $60^{\circ} \mathrm{C}$ for $60 \mathrm{sec}$. GAPDH (mouse) was used as an endogenous control, and relative expression levels of each lncRNA was calculated using the $2^{-\Delta \Delta \mathrm{Cq}}$ method. The primer sequences of investigated lncRNAs are listed in Table I.

Construction of the IncRNA and mRNA co-expression network. The aforementioned validated lncRNAs were used to construct a co-expression network to explore specific mRNAs involved in PA tumorigenesis. The Pearson correlation coefficient (PCC) was calculated for correlations between the validated lncRNAs and all the differentially expressed mRNAs. The lncRNAs and mRNAs with an absolute value of Pearson correlation coefficients $>0.995$ were selected to draw the gene network using the program Cytoscape (version 3.0.1; https://cytoscape.org/).

Statistical analysis. Statistical analyses were performed using SPSS software (version 19.0; IBM Corp., Armonk, NY, USA). For normally distributed data, the differences between two groups were determined using a two-tailed Student's t-test. $\mathrm{P}<0.05$ was considered to indicate a statistically significant difference.

\section{Results}

Overview of IncRNA and mRNA expression profiles. To identify the differentially expressed lncRNAs associated with PA tumorigenesis, the present study examined the expression pattern of lncRNAs in three submandibular tumors from PLAG1 transgenic mice and three normal submandibular samples from control wild-type mice. The expression profiles of the lncRNAs and mRNAs are listed in Table II. The present data demonstrated that $25.36 \%$ of the lncRNAs $(9,110 / 35,923)$ and $31.15 \%$ of the mRNAs $(7,750 / 24,881)$ were significantly differentially expressed in the tumor group as compared with those in the control group $(\mathrm{FC}>2, \mathrm{P}<0.05)$. The unsupervised hierarchical clustering of the top 20 upregulated and downregulated lncRNAs and mRNAs indicated that the expression patterns of the three samples in each group were similar, as indicated by the heat map (Fig. 1). 
Table I. Primer sequences.

\begin{tabular}{|c|c|c|c|}
\hline Primer name & Sequence & $\begin{array}{c}\text { Annealing } \\
\text { temperature }\left({ }^{\circ} \mathrm{C}\right)\end{array}$ & $\begin{array}{l}\text { Product } \\
\text { length (bp) }\end{array}$ \\
\hline GAPDH (mouse) & $\begin{array}{l}\text { F: 5'-CACTGAGCAAGAGAGGCCCTAT-3' } \\
\text { R: 5'-GCAGCGAACTTTATTGATGGTATT-3' }\end{array}$ & 60 & 144 \\
\hline ENSMUST00000140495 & $\begin{array}{l}\text { F: 5'-TCGTGGTGTTTTATCAAGCAAG-3' } \\
\text { R: 5'-ATAGGCTCCGCACTGTTGT-3' }\end{array}$ & 60 & 203 \\
\hline ENSMUST00000125649 & $\begin{array}{l}\text { F: 5'-CGATGACAAGCTGAGGGACA-3' } \\
\text { R: 5'-GCCTTCTTGCTGATCATATTTCTG-3' }\end{array}$ & 60 & 118 \\
\hline AK006017 & $\begin{array}{l}\text { F: 5'-TGACACCGGGGTCTGAGTAT-3' } \\
\text { R: 5'-CCGTAGGTCTGAAATATTCTGC-3' }\end{array}$ & 60 & 210 \\
\hline AK146794 & $\begin{array}{l}\text { F: 5'-TGGATGGTAACAAAAACATGTG-3' } \\
\text { R: 5'-TGAGAGAATGAGGCAAGAGAAG-3' }\end{array}$ & 60 & 65 \\
\hline ENSMUST00000120145 & $\begin{array}{l}\text { F: 5'-AGAAGCTAGTTCTATAGGAAGCAG-3' } \\
\text { R: 5'-CATAGATGTGCTCCAGAAAAAG-3' }\end{array}$ & 60 & 137 \\
\hline AK089826 & $\begin{array}{l}\text { F: 5'-GTGGTACAGTATCGCTAAAGGAC-3' } \\
\text { R: 5'-GGGAAAACATAGTGATAGGAGC-3' }\end{array}$ & 60 & 142 \\
\hline
\end{tabular}

Table II. Summary of the results of lncRNA and mRNA profile analyses.

\begin{tabular}{lcccc}
\hline Probe class & Total, $\mathrm{n}$ & Differentially expressed, $\mathrm{n}(\%)$ & Upregulated, $\mathrm{n}(\%)$ & Downregulated, $\mathrm{n}(\%)$ \\
\hline IncRNAs & 35,923 & $9,110(25.36)$ & $4,521(12.59)$ & $4,589(12.77)$ \\
mRNAs & 24,881 & $7,750(31.15)$ & $4,201(16.88)$ & $3,549(14.26)$ \\
Combined & 60,804 & $16,860(27.73)$ & $8,722(14.34)$ & $8,138(13.38)$ \\
\hline
\end{tabular}

lncRNA, long noncoding RNA.
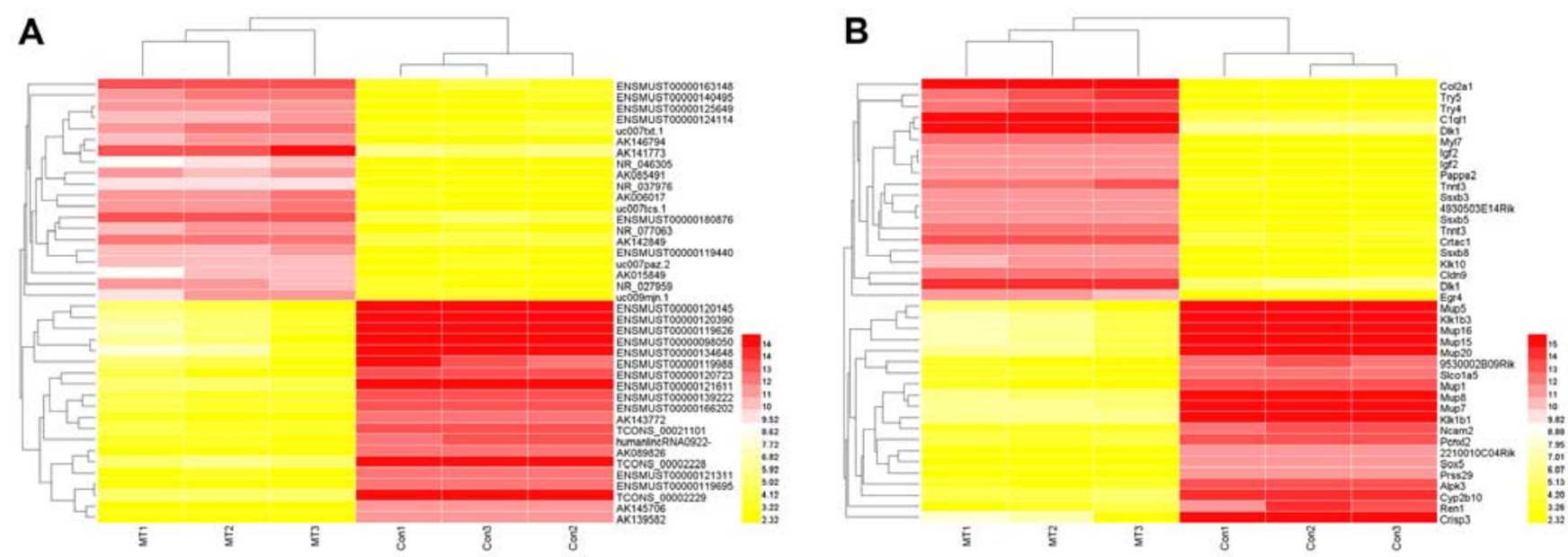

Figure 1. Heatmap of the differentially expressed (A) lncRNAs and (B) mRNAs. In the heatmap, red represents upregulated lncRNAs or mRNAs, while yellow represents downregulated lncRNAs or mRNAs. IncRNA, long noncoding RNA; MT, mouse tumor; Con, control.

Differentially expressed lncRNAs in PLAG1 transgenic mice and wild-type mice. Volcano plot filtering was used to identify the differences in the expression of lncRNAs between the PLAG1 transgenic mice and control mice (Fig. 2A). The plot demonstrated that a total of 9,110 lncRNAs (4,521 upregulated and 4,589 downregulated) were significantly differentially expressed. The chromosome distribution of the differentially expressed lncRNAs was then determined. As illustrated in Fig. 2B, chromosome 2 exhibited the highest number of differentially expressed lncRNAs, while chromosome Y exhibited the lowest number. On the basis of different transcriptional forms, the differentially expressed lncRNAs were 

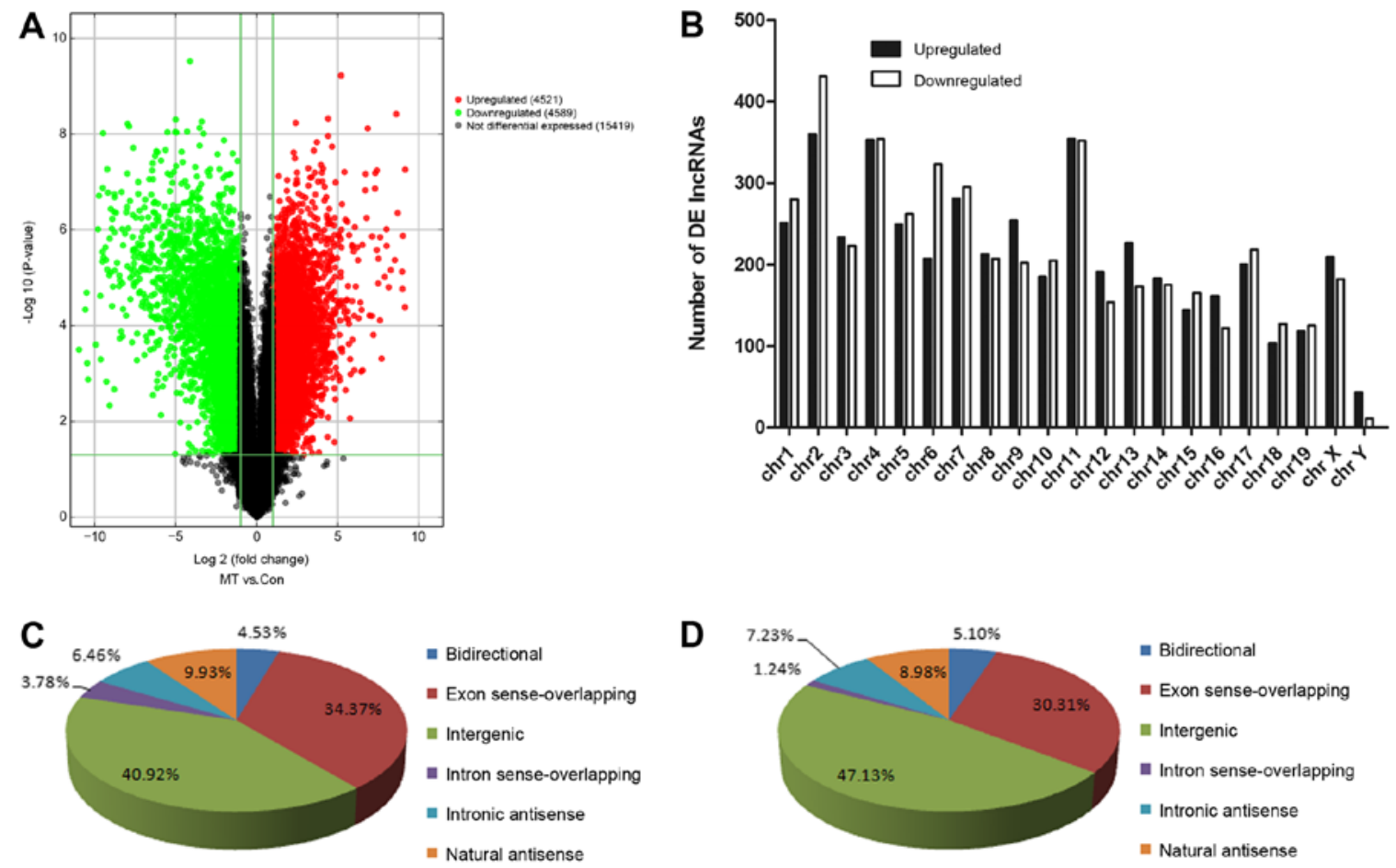

Figure 2. DE lncRNAs in PLAG1 transgenic mice and wild-type mice. (A) Volcano plot for the DE lncRNAs, in which the x-axis represents the FC (log2) and the $y$-axis represents the P-value (-log10). Red points $(\mathrm{FC}>2)$ indicate the upregulated lncRNAs and green points (FC $<-2)$ indicate the downregulated lncRNAs. (B) Chromosomal distribution of the DE lncRNAs. (C) Upregulated and (D) downregulated DE IncRNA classification based on their genomic architecture. lncRNA, long noncoding RNA; DE, differentially expressed; FC, fold change.

A

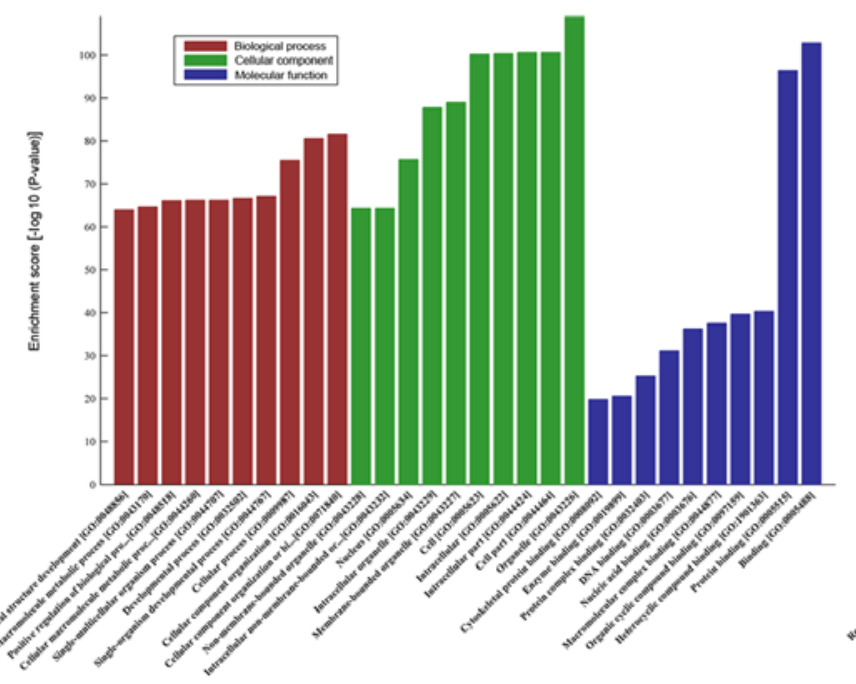

B

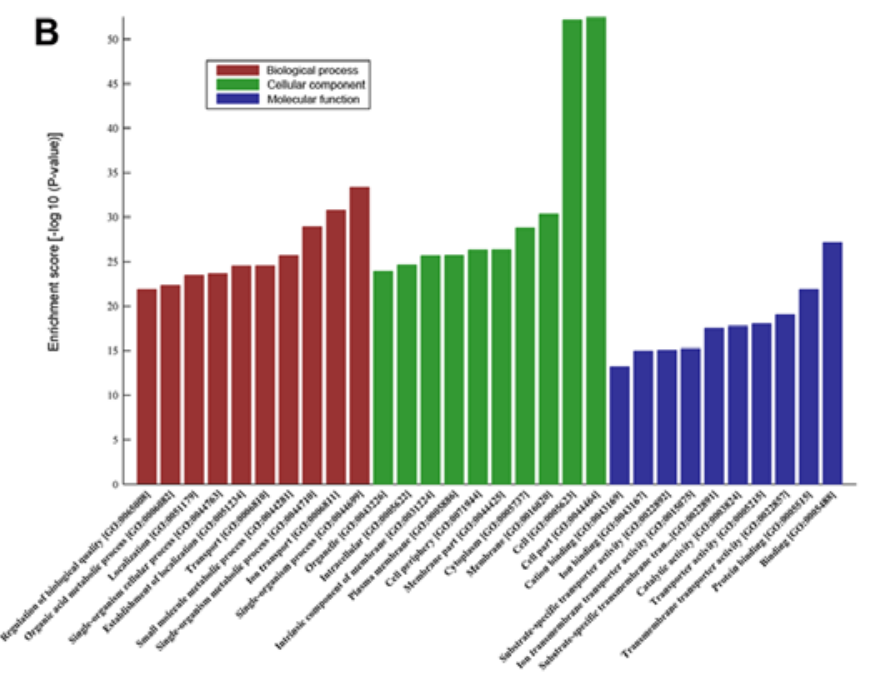

Figure 3. GO term enrichment analysis. GO term enrichment for (A) upregulated and (B) downregulated DE mRNAs. In the GO enrichment analysis, red represents the biological process category, green represents the cellular component, and blue represents the molecular function. DE, differentially expressed; GO, Gene Ontology.

classified into six different subgroups, including bidirectional, exon sense-overlapping, intergenic, intron sense-overlapping, intronic antisense and natural antisense lncRNAs. The majority of the differentially expressed lncRNAs were classified as intergenic and exon sense-overlapping $\ln R \mathrm{NAs}$, both in the upregulated and downregulated groups (Fig. 2C and D).

GO and KEGG pathway analyses of differentially expressed $m R N A s$. GO analysis was conducted to determine the enrichment of the differentially expressed mRNAs in the biological process, cellular component and molecular function categories. The upregulated mRNAs were significantly enriched in a number of GO terms, with the two most prominent molecular function terms being 'binding' and 'protein binding' (GO:0005488 and GO:0005515; Fig. 3A), the most significant biological process term was 'anatomical structure development' (GO:0048856; Fig. 3A) and the most enriched cellular component was 'non-membrane-bounded organelle' (GO:0043228; Fig. 3A). The downregulated mRNAs were also significantly enriched in a number of GO terms, including 'single-organism 

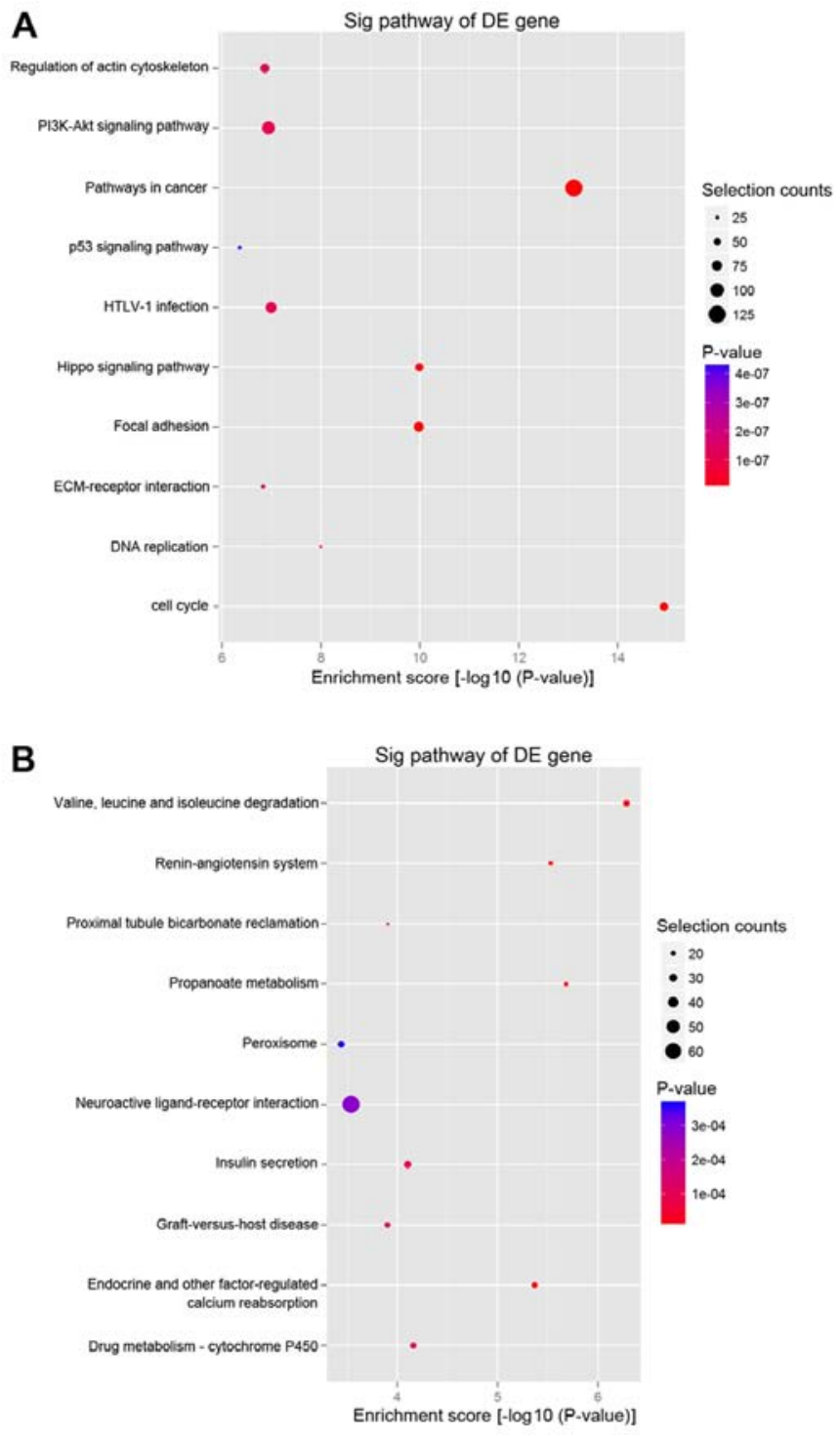

Figure 4. KEGG pathway analysis. The top 10 enriched KEGG pathways are displayed for the (A) upregulated and (B) downregulated differentially expressed mRNAs. KEGG, Kyoto Encyclopedia of Genes and Genomes.

process' (GO:0044699) in biological processes and 'cell part' (GO:0044464) in cellular components (Fig. 3B). In addition, KEGG pathway analysis of the upregulated mRNAs indicated that the two most enriched pathways were 'pathways in cancer' and 'PI3K-Akt signaling pathway' (Fig. 4A), while the downregulated mRNAs were significantly involved in 'valine, leucine and isoleucine degradation' (Fig. 4B).

$R T-q P C R$ validation. To validate the microarray data, RT-qPCR was conducted to detect the expression levels of six most significantly dysregulated lncRNAs (four upregulated and two downregulated) that were selected from the differentially expressed lncRNAs. These selected lncRNAs included ENSMUST00000140495, ENSMUST00000125649, AK006017, AK146794 ENSMUST00000120145 and AK089826. The PCR data were consistent with the microarray analysis results, with a concordance rate of $83.33 \%$ (5/6), supporting the reliability of the microarray data (Fig. 5A). Furthermore, as illustrated
A

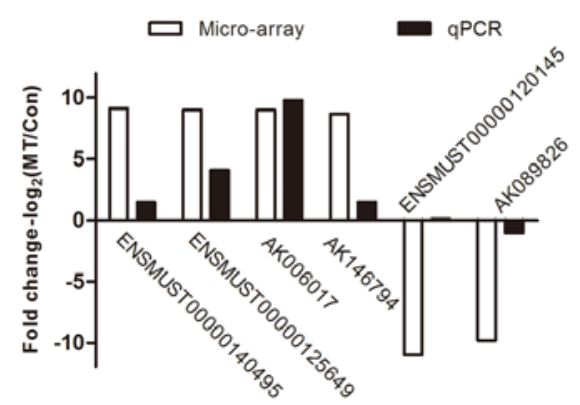

B
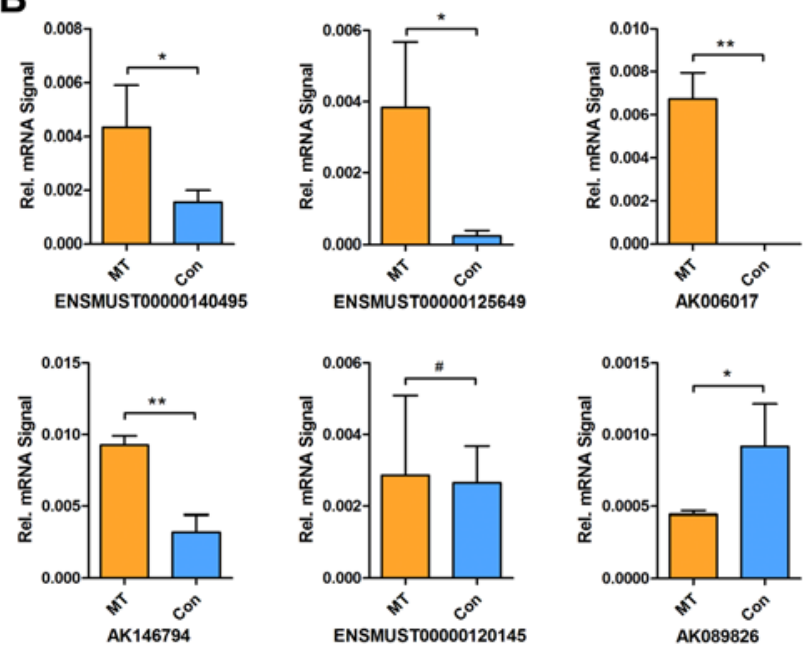

Figure 5. RT-qPCR validation of the 6 selected lncRNAs. (A) Comparison of microarray and RT-qPCR data for differentially expressed lncRNAs. The heights of the columns in the chart represent the log-transformed median fold changes (MT/Con) in expression. (B) Comparison of RT-qPCR data for ENSMUST00000140495, ENSMUST00000125649, AK006017, AK146794 ENSMUST00000120145 and AK089826 in the pleomorphic adenoma tissues obtained from transgenic mice (MT), and the normal gland tissues from wild-type mice (Con). All reactions were performed at least in triplicate $(\mathrm{n}=6) .{ }^{*} \mathrm{P}>0.05 .{ }^{*} \mathrm{P}<0.05$ and ${ }^{* *} \mathrm{P}<0.01$. RT-qPCR, reverse transcription-quantitative polymerase chain reaction; lncRNA, long noncoding RNA; MT, mouse tumor; Con, control; NS, not significant.

in Fig. 5B, RT-qPCR revealed that the expression levels of ENSMUST00000140495, ENSMUST00000125649, AK006017 and AK146794 were significantly increased in the tumor group compared with those in the control group, while AK089826 expression was significantly decreased. By contrast, there was no significant difference in the expression of ENSMUST00000120145 between the tumor and control groups ( $\mathrm{P}>0.05$; Fig. 5B).

Establishment of the IncRNA and mRNA co-expression network. Next, the study attempted to predict the target genes of IncRNAs and to investigate the potential interactions between the IncRNAs and mRNAs in PA in PLAG1 transgenic mice. The associations between the five validated $\operatorname{lncRNAs}$ and corresponding mRNAs were thus assessed by calculating the PCC values. The co-expression network was constructed and visualized using Cytoscape software based on the PCC value (PCC $>0.99$ ). The network illustrated a number of interactions between the five lncRNAs and a total of 725 mRNAs. The network also indicated that a single lncRNA may regulate the mRNA expression of multiple genes, and certain lncRNAs may co-regulate 


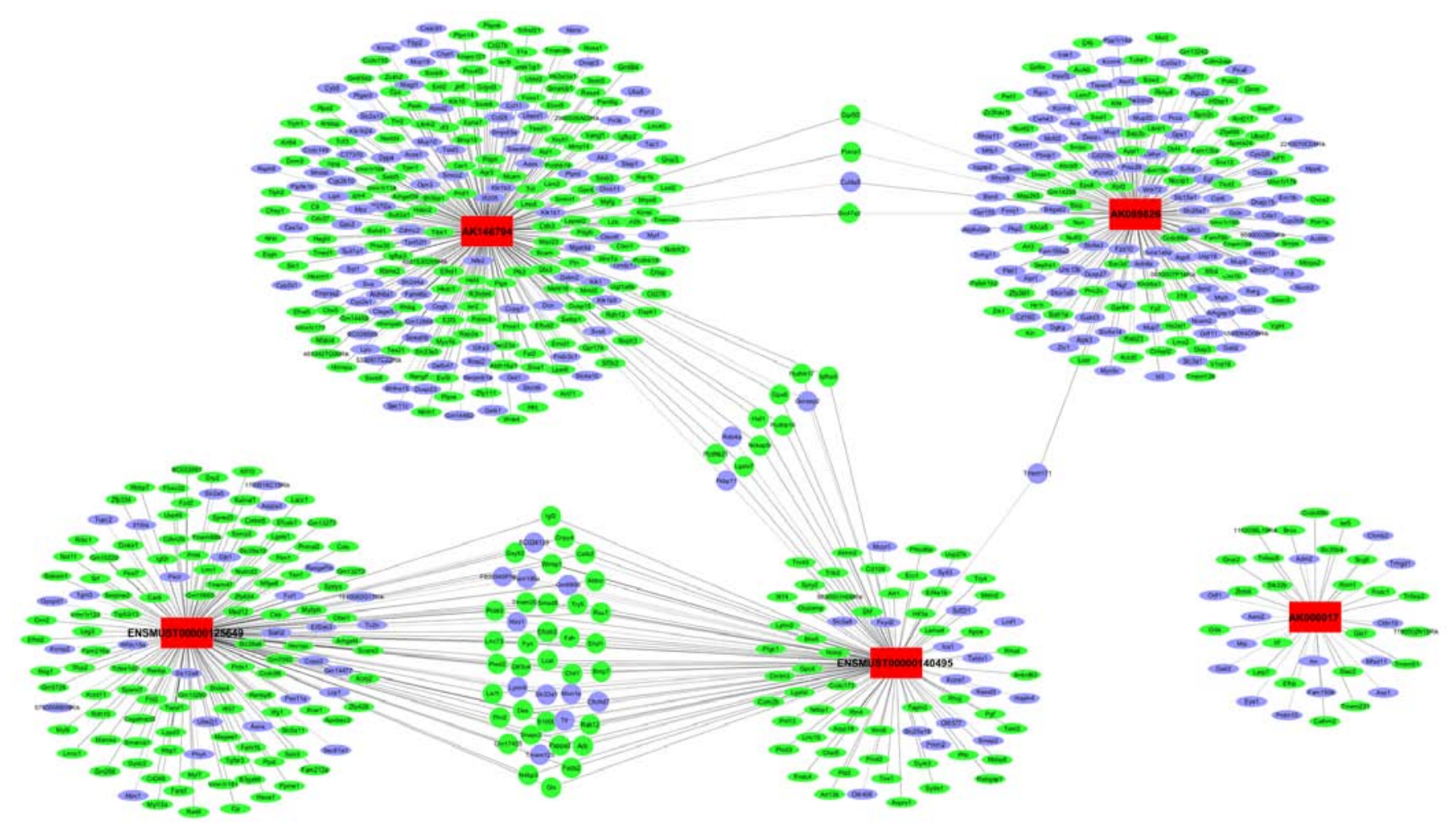

Figure 6. IncRNA and mRNA co-expression network. The red rectangular nodes represent the differentially expressed lncRNAs, the elliptical nodes represent the co-expressed mRNAs, and the round nodes represent the mRNAs co-expressed simultaneously with two or more lncRNAs. The light green color represents upregulated mRNAs and the light blue color represents downregulated mRNAs. IncRNA, long noncoding RNA.

the expression of the same gene. For instance, AK146794 was able to regulate the expression of 264 mRNAs, while IGF2 was simultaneously regulated by ENSMUST00000140495 and ENSMUST00000125649 (Fig. 6).

\section{Discussion}

PA is the most common type of salivary gland tumor with a complex pathogenesis. PLAG1 transgenic mice have been utilized as a useful animal model to study PA. Although a number of genes (including PLAG1, HMGA2 and IGF2) have been proven to be involved in the pathogenesis of PA $(7,21,22)$, it remains unclear how those genes are specifically regulated. With the rapid development of genomic technology, numerous non-coding RNAs (including microRNAs and lncRNAs) have been identified, and their roles in regulating mRNAs are currently researched.

In the present study, the lncRNA expression patterns in submandibular PA tissues of PLAG1 transgenic mice and normal tissues of wild-type C57BL/6 mice were compared using microarray analysis. The data demonstrated that a total of 9,110 lncRNAs were differentially expressed in the submandibular PA tissues obtained from PLAG1 transgenic mice when compared with their counterparts from the wild-type mice, which may facilitate the understanding of the PA-associated global transcriptome. In addition, it was observed that the differentially expressed lncRNAs and mRNAs in PA were distributed unequally among all chromosomes, including the $\mathrm{X}$ chromosome. Notably, all chromosomes were implicated in PA, providing additional evidence that the pathogenesis of PA is relatively complex.
Among the differentially expressed lncRNAs, intergenic lncRNAs comprised the largest category, with 1,850 upregulated and 2,163 downregulated members. Intergenic lncRNAs are conserved across multiple species and may function via various mechanisms, including transcriptional regulation, splicing regulation and post-transcriptional regulation (23). Furthermore, the majority of the validated lncRNAs in the present study belong to the category of intergenic lncRNAs, including ENSMUST00000140495, AK006017, AK146794 ENSMUST00000120145 and AK089826, which is consistent with the universality of intergenic lncRNAs.

To further understand the biological functions and molecular mechanisms of PA-associated mRNAs, GO and KEGG pathway analyses were also performed to identify the biological functions and signaling networks enriched among the differentially expressed mRNAs in the current study. Notably, these mRNAs were involved in multiple pathways associated with PA, including 'PI3K-Akt signaling pathway' and 'p53 signaling pathway'. The PI3K-Akt signaling pathway, a key oncogenic pathway, has been reported to be overactivated in numerous tumors $(24,25)$. The microarray results of the present study indicated that the expression of a number of key molecules of the PI3K-Akt pathway, including IRS1, PI3K, Ras and Akt, was increased. With regard to the p53 signaling pathway, it was observed that p21, Bax, Fas and CyclinD were also activated. However, with respect to intrinsic complex regulatory mechanisms, the specific role of these pathways in PA tumorigenesis warrants further investigation.

Unlike microRNAs, there are no reliable softwares that may be used to predict the target genes of IncRNAs and their functions via their sequence information or secondary 
structure. Thus, the lncRNA-mRNA co-expression network has been developed to predict the potential roles of IncRNAs. In the present study, it was elucidated that the five validated lncRNAs may contribute to the development and progression of PA by altering the expression of target genes. Specifically, the IncRNA ENSMUST00000125649 was observed to be correlated with the IGF2, IGF2R and IGFBP5 mRNAs. In previous studies, it has been demonstrated that PLAG1 overexpression may be responsible for the frequent upregulation of IGF2 $(5,21,22,26)$.

Notably, thousands of dysregulated lncRNAs were identified in the current study, which is possibly due to the influence of PLAG1 overexpression in the transgenic mice. Future studies, particularly functional characterization of these lncRNAs, are thus required.Previous studies have demonstrated that IncRNAs have a wide range of biological functions mediated via various mechanisms, which include cis-targeting, trans-targeting and allosteric modification, and function as enhancers, scaffolds, and co-activators or co-repressors $(23,27)$. A literature search conducted in the current study provided no research results on the aforementioned candidate lncRNAs, which suggests that further research is needed. Additionally, how these functional lncRNAs interact with the associated mRNAs in PA is another issue that merits further study, such as gain- and loss-of-function experiments in vitro, and tumor formation assays in vivo.

In conclusion, the present results demonstrated that a set of lncRNAs were differentially expressed in PA tissues obtained from PLAG1 transgenic mice. These lncRNAs may offer novel insights into the pathogenesis of PA and act as novel therapeutic targets for this tumor.

\section{Acknowledgements}

Not applicable.

\section{Funding}

This study was supported by the National Natural Science Foundation of China (grant no. 81302359) and the Research Grants from the Science and Technology Commission of Shanghai Municipality (grant no. 16ZR1418800).

\section{Availability of data and materials}

The datasets used and/or analyzed during the current study are available from the corresponding author on reasonable request.

\section{Authors' contributions}

SS and WY conceived and designed the experiments. WX conducted RNA sequencing and analysis. LL and HL performed the experiments. WX and $\mathrm{CZ}$ wrote the manuscript. $\mathrm{LL}, \mathrm{JF}$ and $\mathrm{CZ}$ conducted data analysis.

\section{Ethics approval and consent to participate}

All animal experiments were approved by The Ethics Committee of the Faculty of Medicine, Shanghai Jiao Tong University.

\section{Patient consent for publication}

Not applicable.

\section{Competing interests}

The authors declare that they have no competing of interests.

\section{References}

1. Li LJ, Li Y, Wen YM, Liu H and Zhao HW: Clinical analysis of salivary gland tumor cases in West China in past 50 years. Oral Oncol 44: 187-192, 2008.

2. Lima SS, Soares AF, de Amorim RF and Freitas Rde A: Epidemiologic profile of salivary gland neoplasms: Analysis of 245 cases. Braz J Otorhinolaryngol 71: 335-340, 2005 (In Portuguese).

3. Tian Z, Li L, Wang L, Hu Y and Li J: Salivary gland neoplasms in oral and maxillofacial regions: A 23-year retrospective study of 6982 cases in an eastern Chinese population. Int J Oral Maxillofac Surg 39: 235-242, 2010.

4. Hu YH, Zhang CY, Xia RH, Tian Z, Wang LZ and Li J: Prognostic factors of carcinoma ex pleomorphic adenoma of the salivary glands, with emphasis on the widely invasive carcinoma: A clinicopathologic analysis of 361 cases in a Chinese population. Oral surg Oral Med Oral Pathol Oral Radiol 122: 598-608, 2016

5. Zatkova A, Rouillard JM, Hartmann W, Lamb BJ, Kuick R, Eckart M, von Schweinitz D, Koch A, Fonatsch C, Pietsch T, et al: Amplification and overexpression of the IGF2 regulator PLAG1 in hepatoblastoma. Genes Chromosomes Cancer 39: 126-137, 2004.

6. Astrom A, D'Amore ES, Sainati L, Panarello C, Morerio C, Mark J and Stenman G.: Evidence of involvement of the PLAG1 gene in lipoblastomas. Int J Oncol 16: 1107-1110, 2000.

7. Abi Habib W, Brioude F, Edouard T, Bennett JT, Lienhardt-Roussie A, Tixier F, Salem J, Yuen T, Azzi S, Le Bouc Y, et al: Genetic disruption of the oncogenic HMGA2-PLAG1-IGF2 pathway causes fetal growth restriction. Genet Med 20: 250-258, 2018.

8. Juma AR, Grommen SVH, O'Bryan MK, O'Connor AE, Merriner DJ, Hall NE, Doyle SR, Damdimopoulou PE, Barriga D, Hart AH, et al: PLAG1 deficiency impairs spermatogenesis and sperm motility in mice. Sci Rep 7: 5317, 2017.

9. Matsuyama A, Hisaoka M, Nagao $\mathrm{Y}$ and Hashimoto $\mathrm{H}$ : Aberrant PLAG1 expression in pleomorphic adenomas of the salivary gland: A molecular genetic and immunohistochemical study. Virchows Arch 458: 583-592, 2011.

10. Debiec-Rychter M, Van Valckenborgh I, Van den Broeck C, Hagemeijer A, Van de Ven WJ, Kas K, Van Damme B and Voz ML: Histologic localization of PLAG1 (pleomorphic adenoma gene 1) in pleomorphic adenoma of the salivary gland: Cytogenetic evidence of common origin of phenotypically diverse cells. Lab Invest 81: 1289-1297, 2001.

11. Avadhani V, Cohen C and Siddiqui MT: PLAG1: An immunohistochemical marker with limited utility in separating pleomorphic adenoma from other basaloid salivary gland tumors. Acta Cytol 60: 240-245, 2016.

12. Katabi N, Xu B, Jungbluth AA, Zhang L, Shao SY, Lane J, Ghossein R and Antonescu CR: PLAG1 immunohistochemistry is a sensitive marker for pleomorphic adenoma: A comparative study with PLAG1 genetic abnormalities. Histopathology 72: 285-293, 2018.

13. de Brito BS, Giovanelli N, Egal ES, Sánchez-Romero C, Nascimento JS, Martins AS, Tincani ÁJ, Del Negro A, Gondak RO, Almeida OP, et al: Loss of expression of Plag1 in malignant transformation from pleomorphic adenoma to carcinoma ex pleomorphic adenoma. Hum Pathol 57: 152-159, 2016.

14. Zhao X, Ren W, Yang W, Wang Y, Kong H, Wang L, Yan L, $\mathrm{Xu} \mathrm{G}$, Fei J, Fu J, et al: Wnt pathway is involved in pleomorphic adenomas induced by overexpression of PLAG1 in transgenic mice. Int J Cancer 118: 643-648, 2006.

15. Shen S, Yang W, Wang Z, Lei X, Xu L, Wang Y, Wang L, Huang L, Yu Z, Zhang X, et al: Tumor-initiating cells are enriched in CD44(hi) population in murine salivary gland tumor. PLoS One 6: e23282, 2011. 
16. Wang Y, Shang W, Lei X, Shen S, Zhang H, Wang Z, Huang L, Yu Z, Ong H, Yin X, et al: Opposing functions of PLAG1 in pleomorphic adenoma: A microarray analysis of PLAG1 transgenic mice. Biotechnol Lett 35: 1377-1385, 2013.

17. Wang W, Wei C, Li P, Wang L, Li W, Chen K, Zhang J, Zhang W and Jiang G: Integrative analysis of mRNA and lncRNA profiles identified pathogenetic lncRNAs in esophageal squamous cell carcinoma. Gene 661: 169-175, 2018.

18. Li Y, Jin L, Dong A, Zhou X and Yuan H: Microarray expression profile analysis of long non-coding RNAs in optineurin E50K mutant transgenic mice. Mol Med Rep 16: 1255-1261, 2017.

19. Chen Z, Lin S, Li JL, Ni W, Guo R, Lu J, Kaye FJ and Wu L: CRTC1-MAML2 fusion-induced 1ncRNA LINC00473 expression maintains the growth and survival of human mucoepidermoid carcinoma cells. Oncogene 37: 1885-1895, 2018.

20. Shi H, Cao N, Pu Y, Xie L, Zheng L and Yu C: Long non-coding RNA expression profile in minor salivary gland of primary Sjögren's syndrome. Arthritis Res Ther 18: 109, 2016.

21. Declercq J, Van Dyck F, Van Damme B and Van de Ven WJ: Upregulation of Igf and Wnt signalling associated genes in pleomorphic adenomas of the salivary glands in PLAG1 transgenic mice. Int J Oncol 32: 1041-1047, 2008.
22. Voz ML, Agten NS, Van de Ven WJ and Kas K: PLAG1, the main translocation target in pleomorphic adenoma of the salivary glands, is a positive regulator of IGF-II. Cancer Res 60: 106-113, 2000.

23. Ponting CP, Oliver PL and Reik W: Evolution and functions of long noncoding RNAs. Cell 136: 629-641, 2009.

24. Fortin J and Mak TW: Targeting PI3K signaling in cancer: A cautionary tale of two AKTs. Cancer Cell 29: 429-431, 2016.

25. Wang $X$, Zhang X, Wang G, Wang L, Lin Y and Sun F: Hsa-miR-513b-5p suppresses cell proliferation and promotes P53 expression by targeting IRF2 in testicular embryonal carcinoma cells. Gene 626: 344-353, 2017.

26. Akhtar M, Holmgren C, Göndör A, Vesterlund M, Kanduri C, Larsson C and Ekström TJ: Cell type and context-specific function of PLAG1 for IGF2 P3 promoter activity. Int J Oncol 41: 1959-1966, 2012.

27. Ulitsky I and Bartel DP: lincRNAs: Genomics, evolution, and mechanisms. Cell 154: 26-46, 2013.

(c) (i) (-) This work is licensed under a Creative Commons

cc) Atribution-NonCommercial-NoDerivatives 4.0 International (CC BY-NC-ND 4.0) License. 